\title{
NUMERICAL SEISMOGRAMS OF LONG-PERIOD BODY WAVES FROM SEVENTEEN TO FORTY DEGREES
}

\author{
By Donald V. Helmberger
}

\section{ABSTRACT}

Long-period wave propagation in the upper mantle is investigated by constructing synthetic seismograms for proposed models. A model consisting of spherical layers is assumed. Generalized ray theory and the Cagniard-de Hoop method is used to obtain the transient response. Preliminary calculations on producing the phases $P$ and $P P$ by ray summation out to periods of $50 \mathrm{sec}$ is demonstrated, and synthetic seismograms for the long-period World Wide Standard Seismograph Network (WWSSN) and Long Range Seismic Measurement (LRSM) instruments are constructed.

Models containing prominent transition zones as well as smooth models predict a maximum in the $P$ amplitude near $20^{\circ}$. The LRSM synthetics are quite similar for the various models because the instrument is relatively narrow-band, peaked at $20 \mathrm{sec}$. The upper mantle appears smooth at wavelengths greater than $200 \mathrm{~km}$. On the other hand, the WWSSN synthetics are very exciting for models containing structure. The triplications are apparent and the various pulses contain different periods.

The amplitude of the $P$ phase at $30^{\circ}$ is down to about 25 per cent of its $20^{\circ}$ maximum. The amplitude of the $P P$ phase at $35^{\circ}$ is comparable to $P$. Near $37^{\circ}$, the $P P$ phase grows rapidly reaching about twice the $P$ phase amplitude near $40^{\circ}$. Models containing sharp transition zones produce high-frequency interferences at neighboring ranges. A profile of observations is presented for comparison.

\section{INTRODUCTION}

Recent studies on short-period body-wave inversion indicate relatively sharp transition zones in the upper mantle. Such structure produces travel-time triplications, caustics, and a number of delightful propagational effects. Many of these effects have already been discussed with respect to short-period synthetic seismograms (see Gilbert and Helmberger, 1972; Helmberger and Wiggins, 1972; and Müller, 1971). Unfortunately, many of the phase distortions predicted by the synthetics are not obvious in the observations because of the instability of short-period waves. Thus, we would like to use longerperiod motion as a tool in structural exploration as well as in source mechanism studies. This subject is difficult in that there is no available approach at this time that can handle completely broad-band wave propagation. Higher order geometrical optics (Karal and Keller, 1959) has some application at the very short periods, and mode theory treats the longer-period motions. We will examine the middle domain, periods between 1 to 30 secs by the application of generalized ray theory. However, by neglecting millions of rays, our solutions are only rough approximations. We will try to justify the omission of rays by computing simple models for which other solutions are available. Because our technique is a ray theory expansion, it is closely related to geometrical optics and is more accurate at the shorter periods. This relationship with optics is easily seen by considering simple models such as pulse propagation in a homogeneous sphere (see Jeffreys and Lapwood, 1957). Their results indicate that the $P P$ phase behaves like

$$
\frac{1}{\pi} \ln \left|t-t_{p p}\right|
$$

where $t_{p p}$ is the geometrical travel time of the $P P$ phase for a step-function source. The 
change of shape of the seismic pulse can be explained by the fact that $t_{p p}$ is not a true minimum time. The same type of pulse distortion occurs for post-critical angle reflections. The only difference is that in the latter case the reflection coefficient is asymmetric about the reflection time. The symmetry of the $P P$ phase disappears as we introduce velocity gradients in the Earth as we will show. First, we consider the simpler case of wave propagation in a homogeneous sphere.

\section{Homogeneous SPHere}

In this section, we give a brief review of generalized ray theory for a whole space described in spherical coordinates followed by a discussion of wave propagation in a homogeneous sphere.

We assume a point source emitting a spherical wave described by the displacement

$$
U=\frac{\partial}{\partial R} \phi\left(t-\frac{R}{V}\right)
$$

where the displacement potential $\phi$ is defined by

$$
\phi(R, t)=\phi_{o} \frac{H[t-(R / V)]}{R}
$$

and $H$ is the step function, $R$ is the distance between source and receiver in kilometers, and $V$ is the velocity in kilometers per second. $\phi_{o}$ is expressed in cubic kilometers and set equal to unity. This solution is exact for a whole space and indicates no distortion of wave shape with only a $1 / R$ decay. We want to reproduce this result by a rather complicated method to demonstrate the applicability and accuracy of our technique. We start by embedding spherical coordinates into the space (see Figure 1). With this geometry
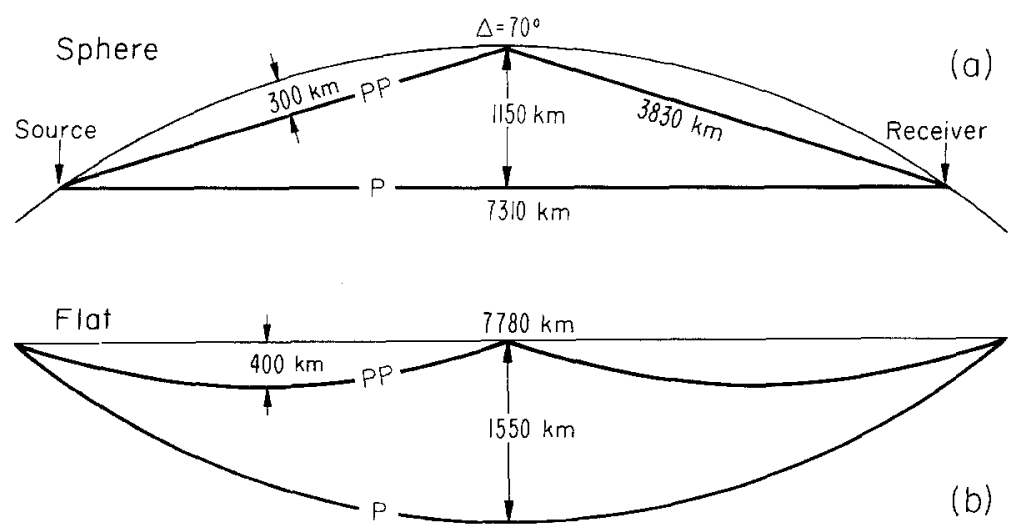

Fig. 1. Diagram of source-receiver geometry in spherical coordinates (a) and the distortion of space after earth stretching (b).

$\phi=1.37 \times 10^{-4} H(t-R / V)$. We next approximate the space by an arbitrary number of homogeneous spherical layers and apply the earth stretching transformation (see Gilbert and Helmberger, 1972 and Helmberger and Harkrider, 1972). That is, for the $j$ th layer,

$$
\begin{aligned}
T h_{j} & =r_{j}-r_{j-1} \\
\tau_{j} & =\left(T h_{j} r_{o} / r_{a j}\right) \\
\alpha_{j} & =\left(V_{j} r_{o} / r_{a j}\right) \\
\beta_{j} & =\left(S_{j} r_{o} / r_{a j}\right) \\
\rho_{j} & =\left(D_{j} r_{o} / r_{a j}\right) \\
r_{a j} & =\left(r_{j}+r_{j-1}\right) / 2
\end{aligned}
$$


where $r_{o}$ is the radius of the Earth and $T h, V, S, D$ and $\tau, \alpha, \beta, \rho$ define the layer thickness, velocities and density before and after transformation, respectively. The velocities and thicknesses of each layer increase with depth as indicated by diagram (b) in Figure 1. We can now produce the $P$ phase by applying generalized ray theory and summing only primaries, rays that suffer only one reflection. The results for a fluid model with $V=6$, $D=1$ are represented in Figure 2.

Plots (a), (b), (c) and (d) of Figure 2 are the displacement potentials for various values of $\tau_{j}$. Plot (e) gives the comparison after a convolution with the Long Range Seismic Measurement (LRSM) instrument response, followed by a derivative with respect to

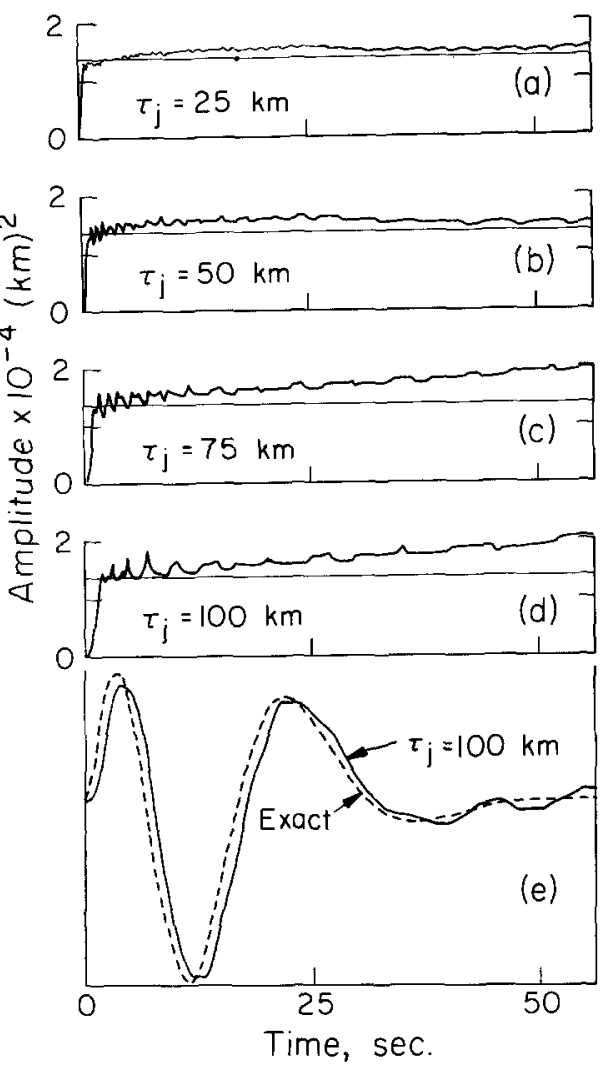

FIG. 2. Comparison of exact solutions (straight lines) with numerical models allowing various layer thicknesses.

time. The corresponding comparisons with Figure 2 (a), (b) and (c) are similar but with less shift and smaller distortions. As the layering thickens the neglect of multiples, rays that suffer more than one reflection, leads to significant errors both at the high - and low-frequency limits as can be seen in Figure 2.

The results with $T h_{j}=50 \mathrm{~km}$ are represented in Figure 3. We varied the density and added a shear velocity. It would appear that changing the density produces neglectable differences. However, the differences between the solid and fluid models is significant. How much of this slow drift is due to neglect of generalized rays containing mixed modes is not known. The number of such rays to sum is prohibitive. On the other hand, the slow drift of the numerical solution can be interpreted as the result of $P$ to $S$ coupling at the very long periods caused by the velocity gradient; that is, the breakdown of the method is due to the earth stretching transformation, as opposed to ray truncation. 
When the responses of Figures 3 are convolved with the LRSM instrument response and compared with the exact response, the results are indistinguishable as demonstrated in plot (d) of Figure 2. Thus, the drift does not seem to be serious for our purposes, although it is interesting.

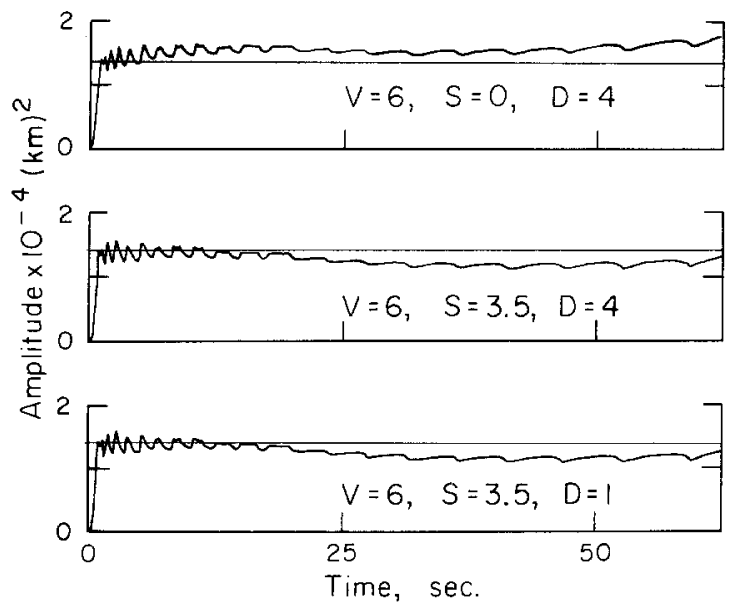

FIG. 3. Comparison of exact solutions (straight lines) with numerical models.

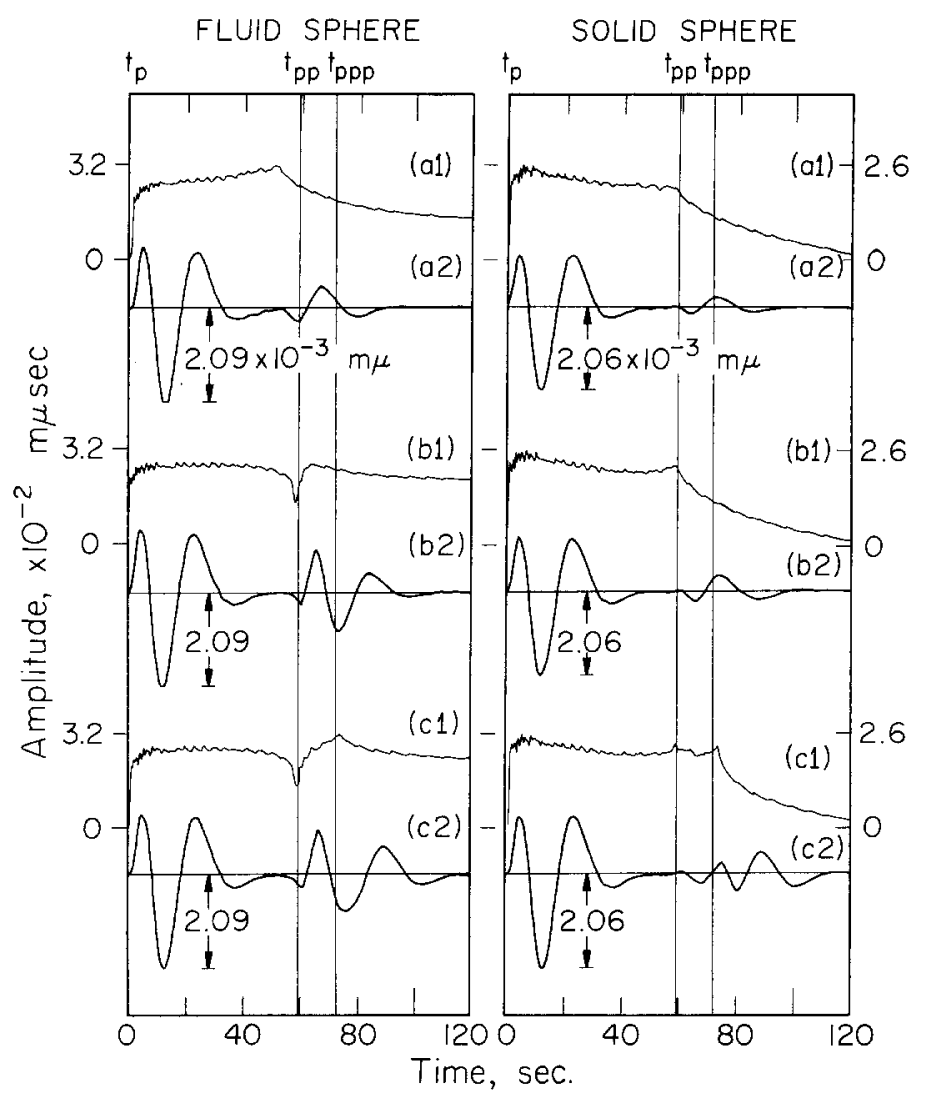

FIG. 4. Vertical displacements and synthetic LRSM responses for a fluid and solid homogeneous sphere at $\Delta=70^{\circ}$. The plots labeled (a1), (b1) and (c1) are the step-function responses after various stages of ray summation. The plots labeled (a2), (b2) and (c2) are corresponding synthetics showing development of the $P P$ phase. 
Using the same source and model except for setting $V=0$ for $r>r_{0}$ and converting potential to vertical displacement, we obtained the results represented in Figure 4 . The plots labeled (a), (b) and (c) in Figure 4 are the time integrals of the displacement at the surface for various stages of ray summation for the cases of a fluid and solid sphere. Using the units defined earlier, one obtains the vertical response in kilometers with source strength, $\phi_{o}$, expressed in cubic kilometers. It is customary to express body waves in units of $m \mu$ and $\phi_{o}$ in cubic meters. Henceforth, in this paper, we assume $\phi_{o}=1 \mathrm{~m}^{3}$ and the synthetic responses expressed in $m \mu$. We will discuss the fluid case first. In Figure 4 plot (a) is the solution after summation of only the primary rays and is, therefore, similar to the results of Figure 2. The largest peak in Figure 4 (a) occurs just before $P P$. Rays contributing to this peak are associated with shallow angle responses from near the surface. Plot (b) of Figure 4 is the solution after the summation of rays that contain 3 reflections including the reflection at the surface, but not containing rays that travel exclusively in the top $200 \mathrm{~km}$ or penetrating deeper than $500 \mathrm{~km}$. Thus, we expect Figure 4 (b) to describe the $P P$ phase. Plot (c) contains the remaining multiples near the surface. The advent of the $P P P$ phase is apparent even though only the first set of multiples was computed. This means that care must be taken in generating synthetics for real earth models so that phases are either well-separated in time or adequately described.

The development of the logarithmic singularity at $t_{p p}$ is somewhat truncated because of numerical technique but should be accurate for computing long-period synthetics. Note that going from Figure 4 (b) to (c) produces a more symmetric $P P$.

The case of a solid with $s=3.5$ is presented in the right-hand column of Figure 4 . The ray summation is performed in the same manner as in the fluid case. Only $P$-mode rays are included. The long-period dip following the $P$ event is caused by the rapid change in the receiver function ( $P$ to $S$ conversion at the surface). The internal reflection coefficient $R_{p p}$ as a function of angle of incidences for the parameter used here is given by Figure 2.3 of Ewing et al. (1957). One sees that $R_{p p}\left(70^{\circ}\right)$ is positive and small, which explains the change of polarity observed in plot (c) of Figure 4 . This feature is discussed by Burridge (1963).

\section{MODELING $P$}

In this section, we return to the problem of computing synthetic seismograms for realistic earth models. We consider two models, the Jeffreys-Bullen model and HWNE (Helmberger and Wiggins, 1971) (see Figure 5). The step-function response, or the time integral of the displacement, and associated LRSM synthetic seismograms for the JB model is presented in Figure 6. Similar plots for the HWNE model are presented in Figure 7; also included is the World Wide Standard Seismograph Network (WWSSN) synthetic seismograms. The effect of absorption $(Q=500)$ on the step-function response and synthetics is given in Figure 8.

A small triplication occurs for the JB model near $20^{\circ}$. This feature is produced by a change in slope of the velocity structure at a depth of $413 \mathrm{~km}$. The effect on pulse shape is displayed in Figure 6 . Note that the distortion in the long-period motion persists well past the geometrical cutoff of the triplication which is about $21.5^{\circ}$. These extensions show a depletion at the shorter periods as they must to obey the zero wavelength limit. The step-function response for model HWNE indicates even more frequency-dependent effects because the structure is more detailed. Although these models in fine structure are quite different, their large scale features are similar and their LRSM synthetics are remarkably alike. The only large scale difference between the two models is the $660-\mathrm{km}$ transition zone. This feature tends to spread out the $19^{\circ}$ maximum that is so prevalent 


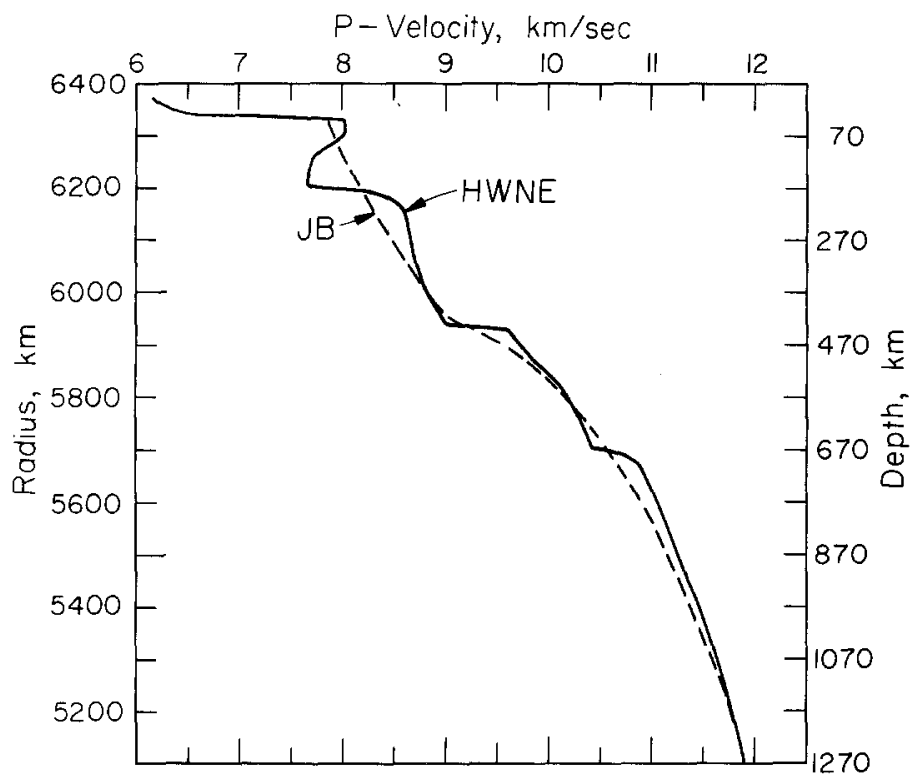

Fig. 5. Upper-mantle models.

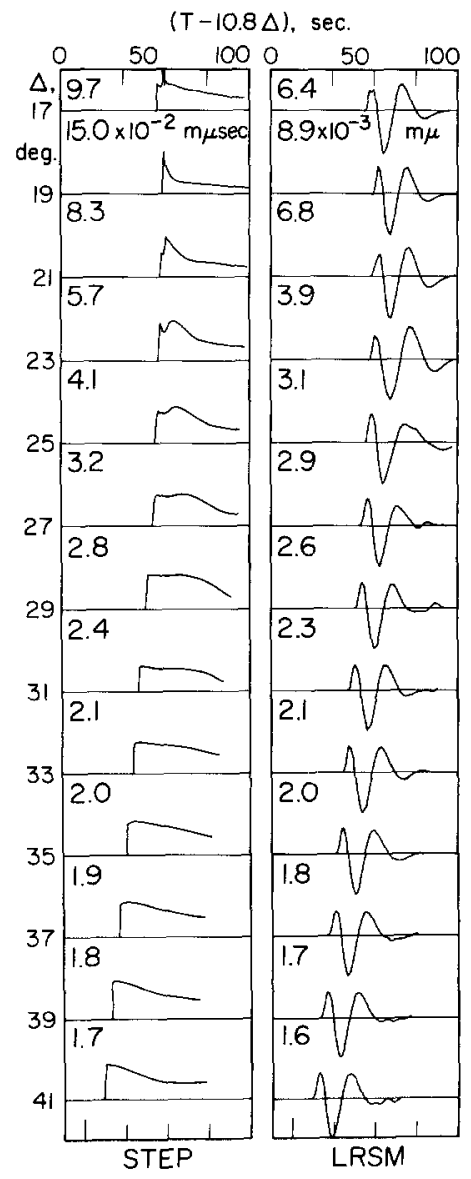

Fig. 6. Step-function response and synthetic seismogram profile assuming the Jeffreys-Bullen model. Peak amplitude for each trace is indicated on plots. 
in the JB model. At the larger ranges, the synthetics are similar except that the JB model has a slightly higher velocity gradient at depth which tends to enhance the amplitude.

The most interesting features of these synthetics is the performance of the WWSSN instrument. It seems that most of the significant details predicted by the step-function response is maintained after convolution (see Figure 7). Even the frequency differences between arrivals is apparent, as in the synthetic at $17^{\circ}$. It is interesting to examine these synthetics after the high frequencies have been removed either by a more realistic source

$(T-10.8 \Delta)$, sec.

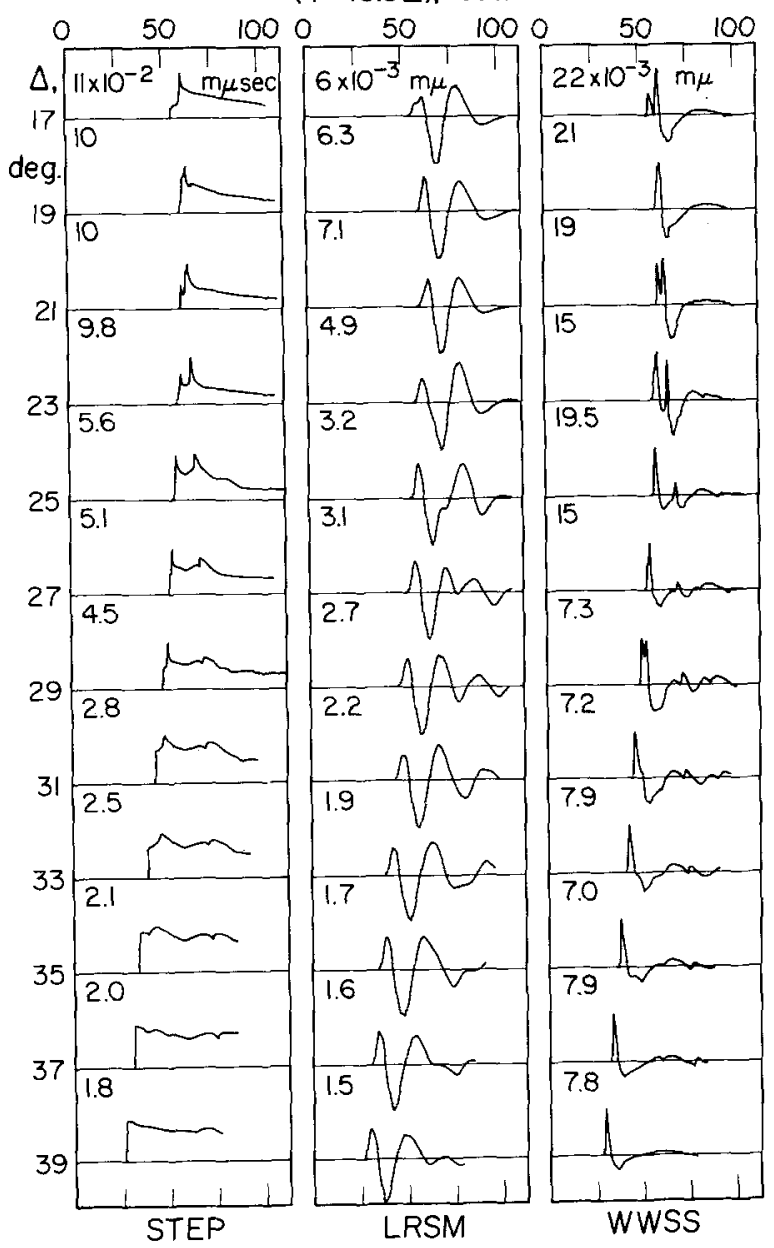

FIG. 7. Step-function response and synthetic seismogram profiles assuming the HWNE model. LRSM and WWSSN (15-100) instrument responses used. Peak amplitude for each trace is indicated on plots.

or by absorption. This can be accomplished by assuming a " $Q$ " filter (Carpenter, 1967), (see Figure 8 ). The results are similar to Figure 7 except that it becomes more difficult to observe the triplications.

It was noticed in comparing the amplitudes of short-period synthetics with the corresponding ( $p$ versus $\Delta$ ) plots (see Wiggins and Helmberger, 1972) that a rather large amount of flexibility existed in fitting the relative amplitudes of two arrivals. The reason for this is the interaction of the step-function response with the short-period instrument, $S P$; that is, one cannot tell the difference between a sharp small spike from a larger but smoother peak. Examples of peaks having diverse frequency content are apparent in 
Figures 6,7,8. However, it is true that complete coverage of $S P$ 's allows one to distinguish between sharp boundaries and caustics (see Helmberger and Wiggins, 1971). The point is that one broad-band observation such as those in Figures 7 and 8 contains information about the shapes of structure. This means that fewer observations allow the same degree of structural determination. Actually, the addition of other unknowns, such as scattering and absorption, puts some strong constraints on inversion based on frequency content. Furthermore, inasmuch as the density of WWSSN stations is so low compared to the

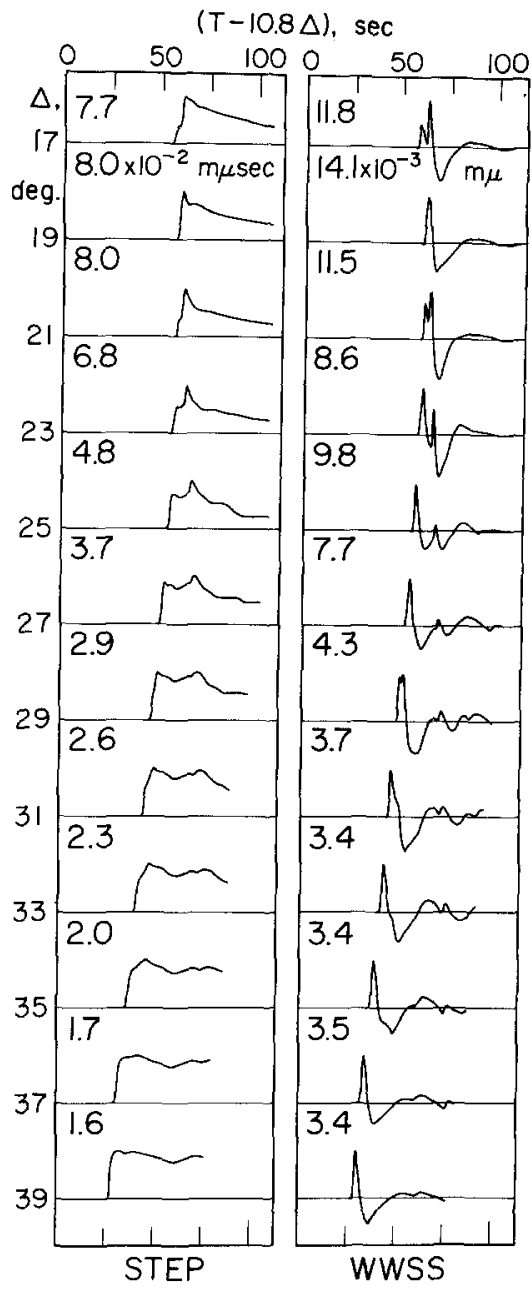

Fig. 8. Step function and synthetics after applying $Q$ operator $(Q=500)$. Peak amplitude for each trace is indicated on plots.

former LRSM network, one would expect composite studies to be the most rewarding. The determination of $Q$ structure may be possible by this type of approach.

It is interesting to make a brief comparison of the synthetics of Figure 8 with some observations from a relatively simple earthquake presented in Figure 9. It seems that the second pulse at FSJ is coming from the $440-\mathrm{km}$ transition. The first arrival at YKC could be coming from the $660-\mathrm{km}$ transition and the second pulse, from the $440-\mathrm{km}$ transition. These two arrivals seem to cross at $19^{\circ}$ as in the synthetics. The double arrival at CMC looks much like the synthetics at $29^{\circ}$. However, this rather free interpretation is only speculative, because we have not included the radiation pattern nor the effects of $p P$ and $s P$. An attempt at such modeling will be reported on later. 


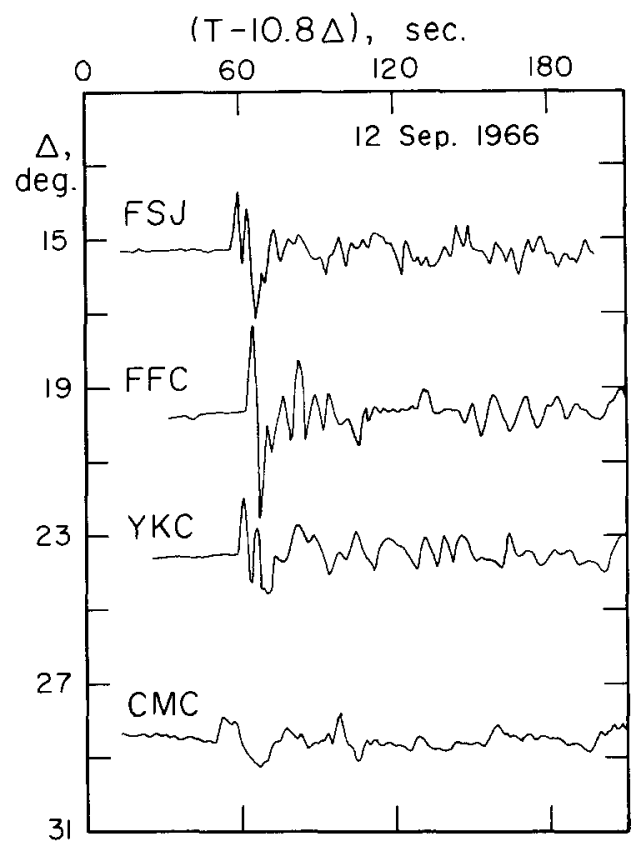

FIG. 9. Observations from the Truckee, California, earthquake of September 12, 1966.

\section{MODELING $P P$}

There has been considerable effort made at explaining the behavior of the $P P$ phase both from observational and theoretical points of view. One of the first observational studies was made by Gutenberg and Richter (1935). They demonstrated that the ratio $(P P / P)$ has a maximum near $40^{\circ}$ and that this maximum seems to have regional behavior, continental and oceanic paths being different. Byerly et al. (1949) pointed out the difficulty with this ratio measure, namely, the vertical radiation pattern effects. This explanation probably explains the rather large scatter in their data. We attempted in this study to minimize this effect by choosing stations along constant azimuth and examining the radiation pattern to make sure the observations are away from a node. A map of stations used and the vertical radiation pattern is presented in Figure 10. The fault-plane solution was determined in the conventional manner; the vertical radiation pattern, after Ben Menahem et al. (1965), is presented in Figure 10. The observations are given in Figure 11. These wave forms are on the same amplitude scale, corrected only for instrumental gain. The radiated amplitudes for the phases $P, P P, p P$ and $p P P$, appropriate for an observation at $40^{\circ}$, are indicated in Figure 10 . The domination by $p P$ is apparent. It seems that $p P P$ increases its amplitude by a factor of 2 near $40^{\circ}$ and that the ratio of $P P / P$ is slightly less than two. We will attempt to model these effects by applying the technique discussed earlier.

We would like to assume a detailed model of upper-mantle structure and compute a broad-band synthetic over the first few hundred seconds. Because this is too difficult, we assume a simplified model and suppose that the LRSM long-period instrument will perform the proper smoothing. The model is given in Table 1 . We assume a small number of layers so that multiples can be included. One of the advantages of using coarse layering is the ability to label individual pulses as presented in Figure 12. The numbers indicate the reflection time for the various rays; for example, the index 8 refers to the ray that reflects off the bottom of layer 8 . The index 828 refers to the ray that reflects off the 
bottom of 8 and returns to the surface, etc. Note that the index 8 refers to the large jump in velocity associated with the $655-\mathrm{km}$ transition and similarly the index 6 refers to the $425-\mathrm{km}$ transition. The internal multiples are not labeled, but we included all rays that encountered three reflections anywhere in the stack. We allow only $P$ mode rays as before.

The $P$ wave is obviously small because the model does not include that part of the mantle where the geometrical ray would bottom. The model was chosen to predict the
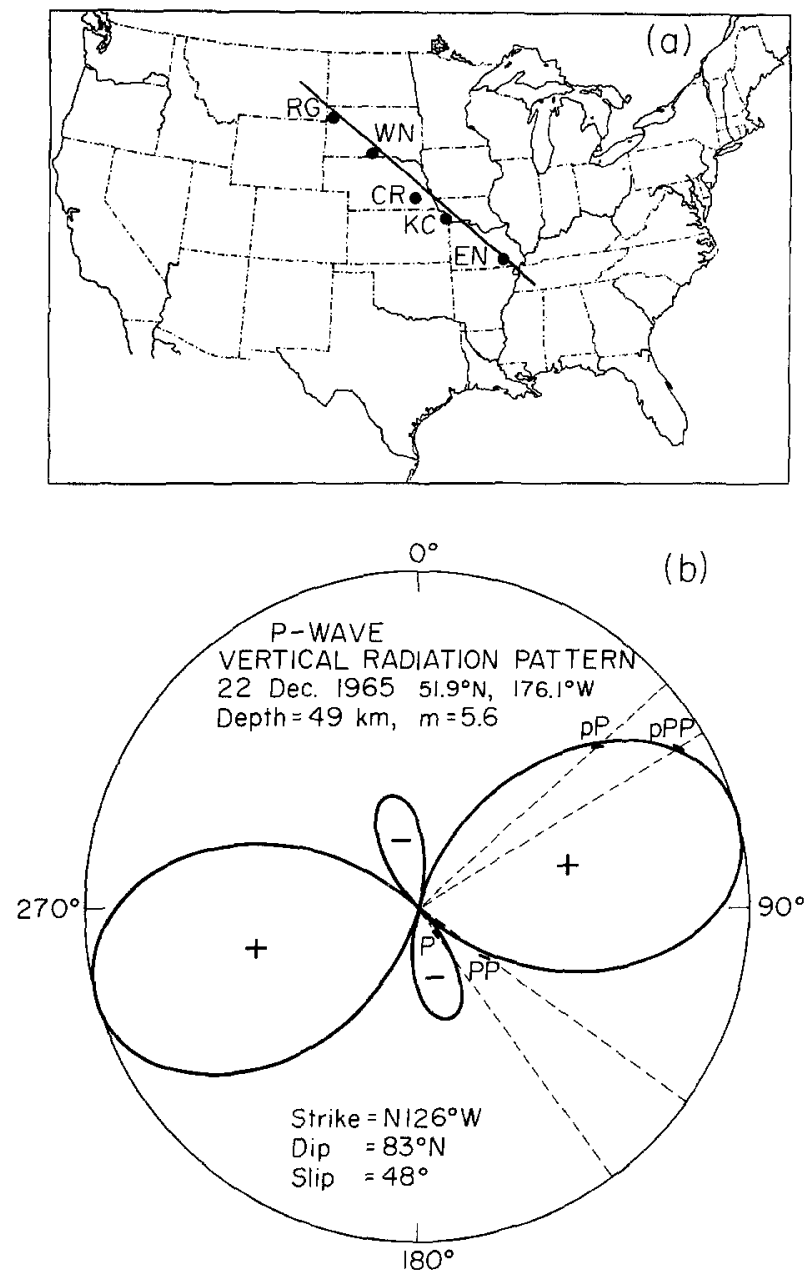

FIG. 10. Locations of LRSM stations (a) along nearly constant azimuth and vertical radiation pattern (b).

$P P$ phase. We will use the synthetic $P$ waves predicted by HWNE as our amplitude criterion when comparing $P$ with $P P$. As a preview, note that the ratio of $P P / P$, using the $P P$ of Figure 12 and the $P$ of Figure 7, yields a value of about 1.5. The $P P$ phase in Figure 12 looks much like the fluid case in Figure 3 (b), except that it is more asymmetrical. This effect is caused by the rapid increase in velocity near the $P P$ geometrical bottoming depth.

A more complete profile of step function and synthetic responses for this model is presented in Figure 13. The spikes can be recognized by the labels presented in Figure 12. They do not seem to be particularly important except $P_{6}$ which creates a significant pulse in the synthetic seismograms between 27 and $33^{\circ}$. This feature also shows up in more realistic earth models, as can be seen in Figure 7. 
One of the motivations for computing this model was to test the significances of multiples and to see how much interference one can expect between $P$ and $P P$ while the latter is developing. The amplitudes taken from Figures 7 and 13 are plotted in Figure 14. It seems that $P$ dominates $P P$ for ranges less than $29^{\circ}$. This may not be the case for shortperiod synthetics but in that case they are well separated in time. At larger ranges, 828 and 626 begin to interfere, producing a large $P P$ near $39^{\circ}$. It should be realized that the JB

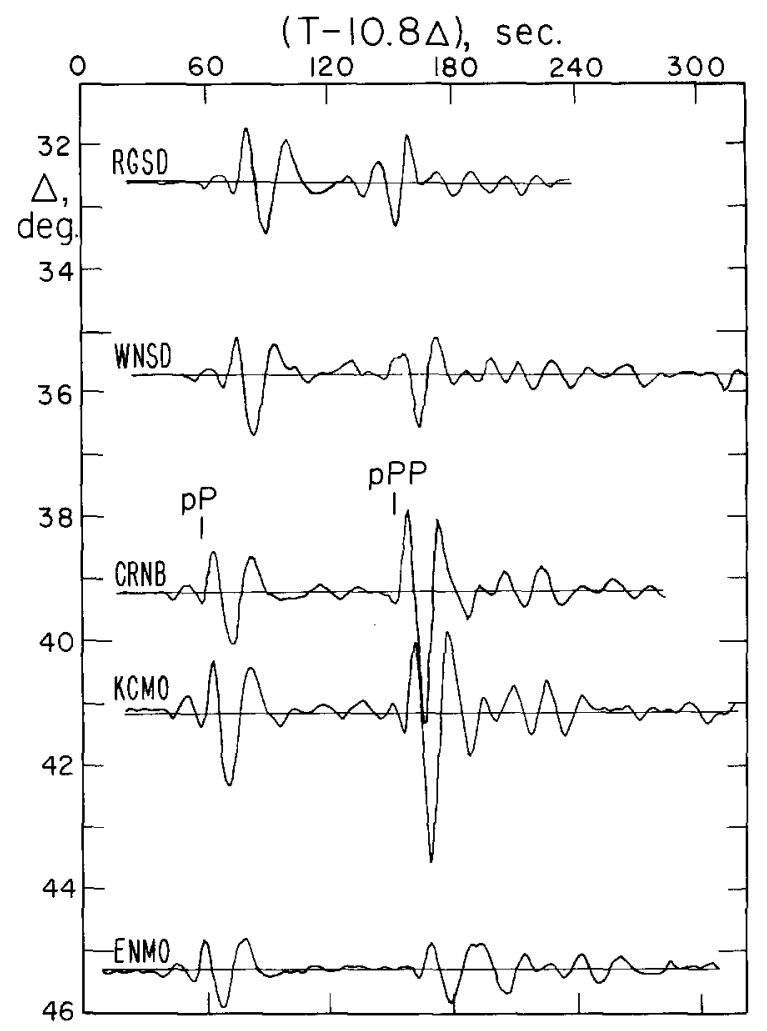

FIG. 11. Vertical long-period LRSM observations from the December 22, 1965 event.

TABLE 1

UPPER MANTLE Model

\begin{tabular}{lcccc}
\hline Layer & $\begin{array}{c}\text { Thickness } \\
(\mathrm{km})\end{array}$ & $\begin{array}{c}\alpha \\
(\mathrm{km} / \mathrm{sec})\end{array}$ & $\begin{array}{c}\beta \\
(\mathrm{km} / \mathrm{sec})\end{array}$ & $\begin{array}{c}\rho \\
\left(\mathrm{gm} / \mathrm{cm}^{3}\right)\end{array}$ \\
\hline & & & & \\
1 & 0 & 0.0 & 0.0 & 0.0 \\
2 & 30 & 6.5 & 3.5 & 2.8 \\
3 & 90 & 8.0 & 4.5 & 3.2 \\
4 & 80 & 7.9 & 4.4 & 3.2 \\
5 & 100 & 8.8 & 4.8 & 3.5 \\
6 & 125 & 9.2 & 5.0 & 3.6 \\
7 & 100 & 10.6 & 5.6 & 4.0 \\
8 & 130 & 11.6 & 5.8 & 4.2 \\
9 & $\infty$ & 13.3 & 6.3 & 4.4 \\
& & & & \\
\hline
\end{tabular}




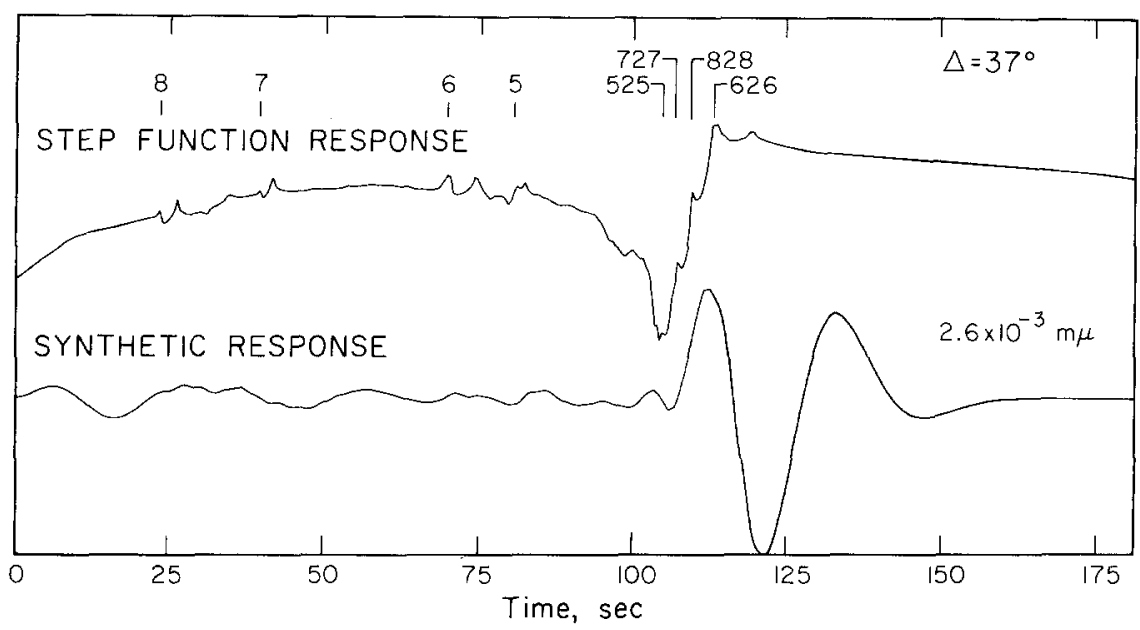

FIG. 12. Synthetic response at $\Delta=37^{\circ}$ for the model given in Table 1 .

$(T-10.8 \triangle), \sec$
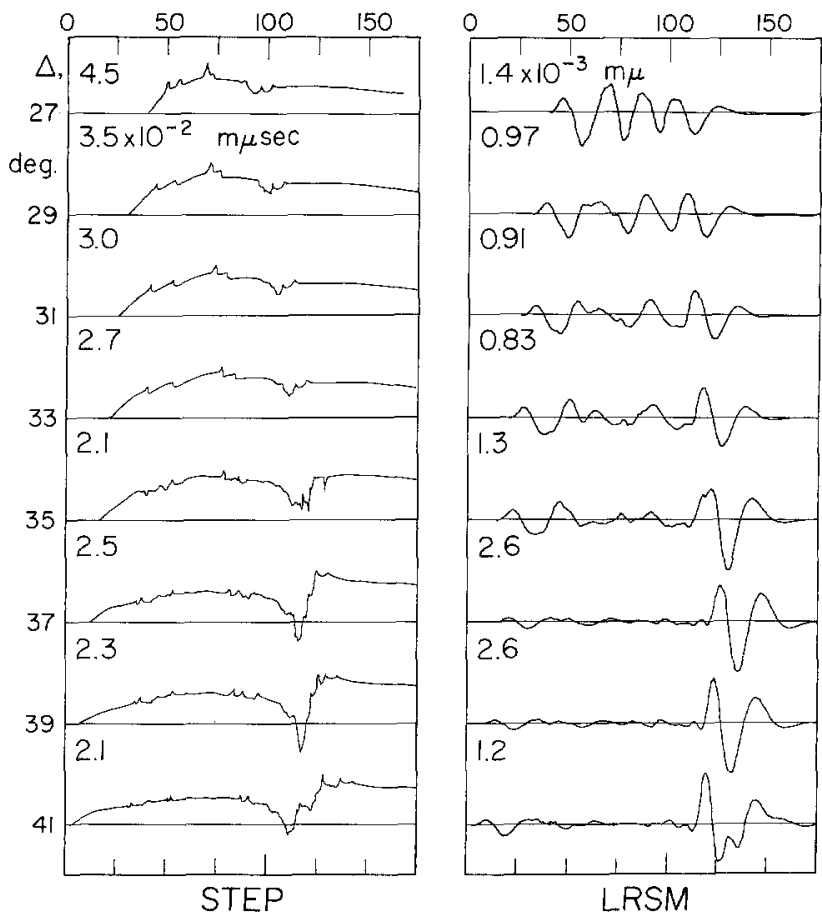

FIG. 13. Synthetic profile based on the model given in Table 1. Peak amplitude for each trace is indicated on plots.

model would also produce a large $P P$ near $40^{\circ}$ because $P$ peaks at $20^{\circ}$. The differences between models would be dramatized by the changes in the $P P$ wave shape. From Figure 13, we would expect to see significant interferences between 35 and $45^{\circ}$. Similarly, we would expect complications in the $P P P$ phase near $60^{\circ}$. The comparatively short-period signals labeled $P_{a}$ by Caloi (1954) could well be such interference effects. Båth and Lopez Arroyo (1963) suggest rather large variations in upper-mantle structure based on $P_{a}$ 
velocity. Their interpretation may be somewhat oversimplified if our interpretation is correct. Long-period array measurements should easily resolve the question.

Comparing our synthetic results with Figure 11, we find good agreement except that the observed $P P$ peaks at slightly larger ranges. This feature is caused by our velocity model, which is somewhat fast. It would seem that the observed $P P$ at $45^{\circ}$ is complicated by the interference mentioned earlier. The $P_{c} P$ phase is missing in this profile, as expected from the vertical radiation pattern represented in Figure 10.

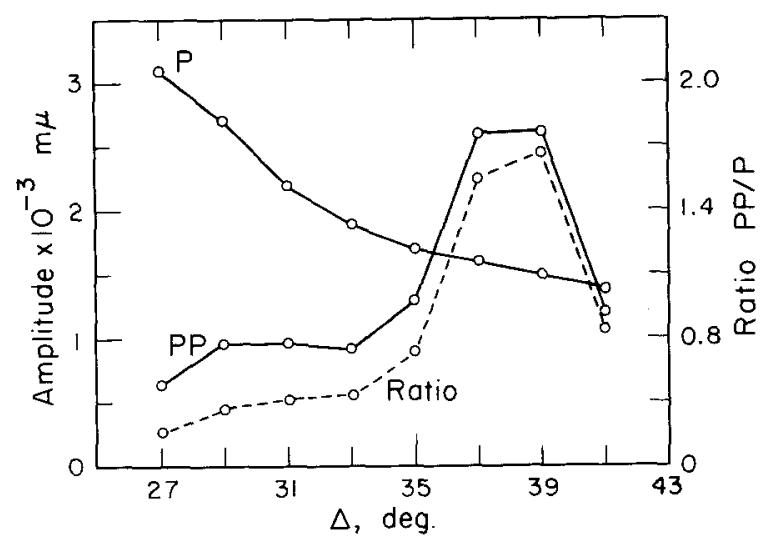

FiG. 14. Synthetic amplitude decay for the phases $P$ and $P P$ and ratio $P P / P$.

\section{CONCLUSION}

A comparison of synthetic responses constructed by the application of generalized ray theory with exact results demonstrates the applicability of our technique to longperiod seismological problems. The method is useful both as an exploration tool and a method for studying source mechanisms. The latter study especially requires broad-band information, which the combination of generalized ray theory and Cagniard's method provides.

Synthetic profiles for the $J B$ and HWNE models were constructed assuming a stepfunction source in displacement potential. Strong interference occurs at ranges where the $P$ phase bottoms near a major transition zone. These profiles suggest the use of long-period WWSSN recordings in structural modeling.

A comparison was made between a LRSM synthetic profile of $P P$ with a similar set of observations. The LRSM observations are easy to justify but are not sensitive enough for modeling purposes. However, one should be able to determine the polarity of unknown source functions with no difficulty. The strong interference that shows up in synthetic $P$ profiles near $20^{\circ}$ is also apparent in $P P$ near $40^{\circ}$. Broad-band array measurements at the appropriate ranges should prove interesting.

\section{ACKNOWLEDGMENTS}

This research was supported by the Advanced Research Projects Agency of the Department of Defense and was monitored by the Air Force Office of Scientific Research under Contract F44620-72-C-0078. 


\section{REFERENCE}

Båth, M. and A. Lopez Arroyo (1963). Pa and Sa waves and the upper mantle, Geofis. Pura Appl. 56, $67-92$.

Ben-Menahem, A., S. T. W. Smith, and T-L. Teng (1965). A procedure for source studies from spectrums of long-period seismic body waves, Bull Seism. Soc. Am. 55, 203-235.

Burridge, R. (1963). The reflexion of a pulse in a solid sphere, Proc. Roy. Soc. London, Ser. A. 276, $367-400$.

Byerly, P., A. I. Mei, S. J. and C. Romney (1949). Dependence on azimuth of the amplitudes of $\boldsymbol{P}$ and PP, Bull. Seism. Soc. Am. 39, 269-284.

Caloi, P. (1954). L'astonesfera come canale-guida dell' energia sismica, Ann. Geofis. 7, 491-501.

Carpenter, E. W. (1967). Teleseismic signals calculated for underground, underwater, and atmospheric explosions, Geophysics 32, 17-32.

Ewing, M. W., W. S. Jardetzky, and F. Press (1957). Elastic waves in layered media, McGraw-Hill, New York.

Gilbert, F. and D. V. Helmberger (1972). Generalized ray theory for a layered sphere, Geophys. J. 27, $57-80$.

Gutenberg, B. and C. F. Richter (1935). On seismic waves, (2nd paper), Beitr. Geophysik. 45, 280-360.

Helmberger, D. and R. A. Wiggins (1971). Upper mantle structure of Midwestern United States, J. Geophys. Res. 76, 3229-3245.

Helmberger, D. and D. G. Harkrider (1972). Seismic source descriptions of underground explosions and a depth discriminate, Geophys.J. 31, 45-66.

Jeffreys, H. and E. R. Lapwood (1957). The reflexion of a pulse within a sphere, Proc. Roy. Soc. London, Ser. A. 241, 455-479.

Karal, F. C., Jr. and J. B. Keller (1959). Elastic wave propagation in homogeneous and inhomogeneous media, J. Acoust. Soc. Am. 31, 694-705.

Müller, G. (1971). Approximate treatment of elastic body waves in media with spherical symmetry, Geophys. J. 23, 435-450.

Wiggins, R. A. and D. V. Helmberger (1972). Upper mantle structure of Western United States, J. Geophys. Res. (in press).

SEISMOLOGICAL LABORATORY

Division of Geologtcal and Planetary Sciences

CALIFORNIA INSTITUTE OF TeChNOLOGY

Pasadena, California

CONTRIBUTION No. 2189

Manuscript received September 14, 1972 